\title{
Acknowledging contributions to online expert assistance
}

Andra Waagmeester Department of Bioinformatics - BiGCaT, Maastricht University, Maastricht, the Netherlands Gareth Palidwor Ottawa Hospital Research Institute, Ottawa, Ontario, Canada Pawel Szczesny Department of Bioinformatics, Institute of Biochemistry and Biophysics, Warsaw, Poland Istvan Albert Bioinformatics, Pennsylvania State University, USA

Mary Mangan OpenHelix LLC, Bellevue, Washington USA

Chris Miller The Genome Institute at Washington University, St. Louis, Missouri, USA Simon Cockell Bioinformatics Support Unit, Newcastle University, Newcastle upon Tyne, UK

Pierre Lindenbaum INSERM, UMR915, Institut du Thorax, Nantes, France Daniel A M M Silvestre LIM01 - Laboratório de Informática Médica - HCFMUSP, São Paulo, SP - Brasil Giovanni Marco Dall'Olio Institute of Evolutionary Biology (UPF-CSIC), CEXS-UPF-PRBB, Barcelona, Spain Chris Evelo Department of Bioinformatics - BiGCaT, Maastricht University, Maastricht, the Netherlands 
File Edit Data View Help

\begin{tabular}{|c|c|c|c|c|c|c|c|c|c|c|}
\hline [承 & \begin{tabular}{l|l} 
& 國
\end{tabular} & 䧄 & (1) & 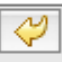 & Zoom: & $115.7 \%$ & $\nabla$ & Gitit? & $\square$ & \\
\hline
\end{tabular}

Title: New Pathway

$7410: \mathrm{BDH} 2$

TP53INP2

P00196

뭄acetyl-L-carnitine뭄
Objects

Properties

Backpage

Data Nodes

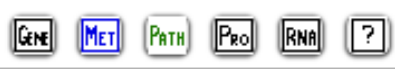

Basic Shapes

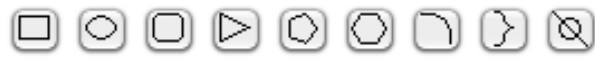

Basic interactions

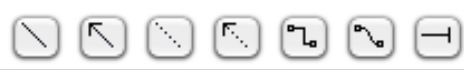

MIM interactions

$\mapsto \rightarrow \rightarrow \rightarrow \oplus \rightarrow \oplus \rightarrow$

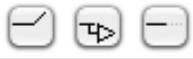

Cellular compartments

(10) 8 (

Annotations

Labet

Templates

道 器 
*OPS.gpml - PathVisio 2.0.11

File Edit Data View Help

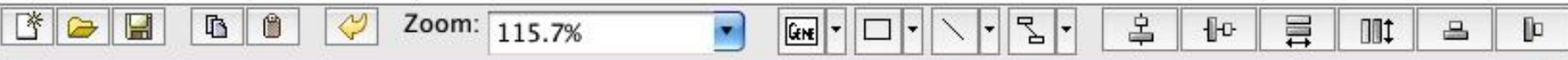

Title: New Pathway

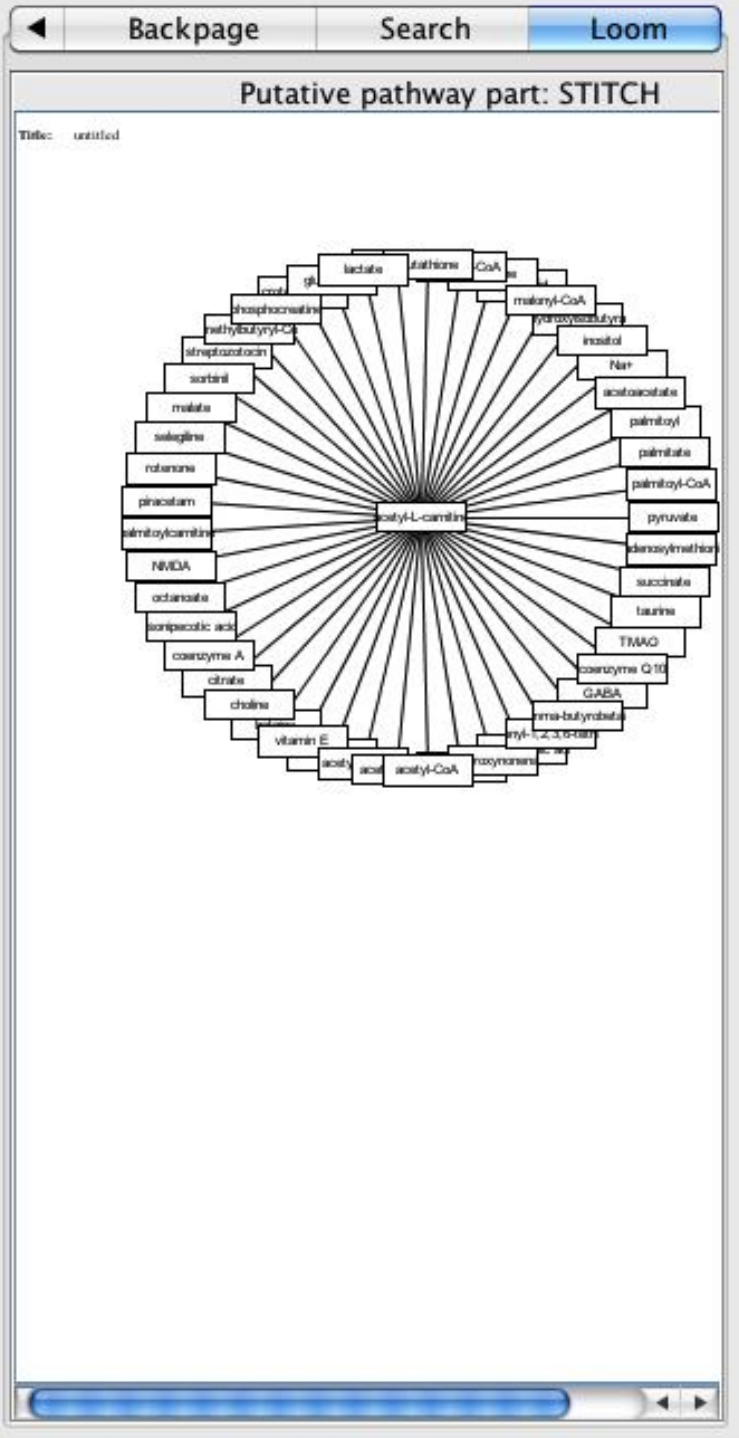

| Gene database: ...s_Derby_20100601.bridge | Metabolite database: ...metabolites_081205.pgdb 


\section{Error!}

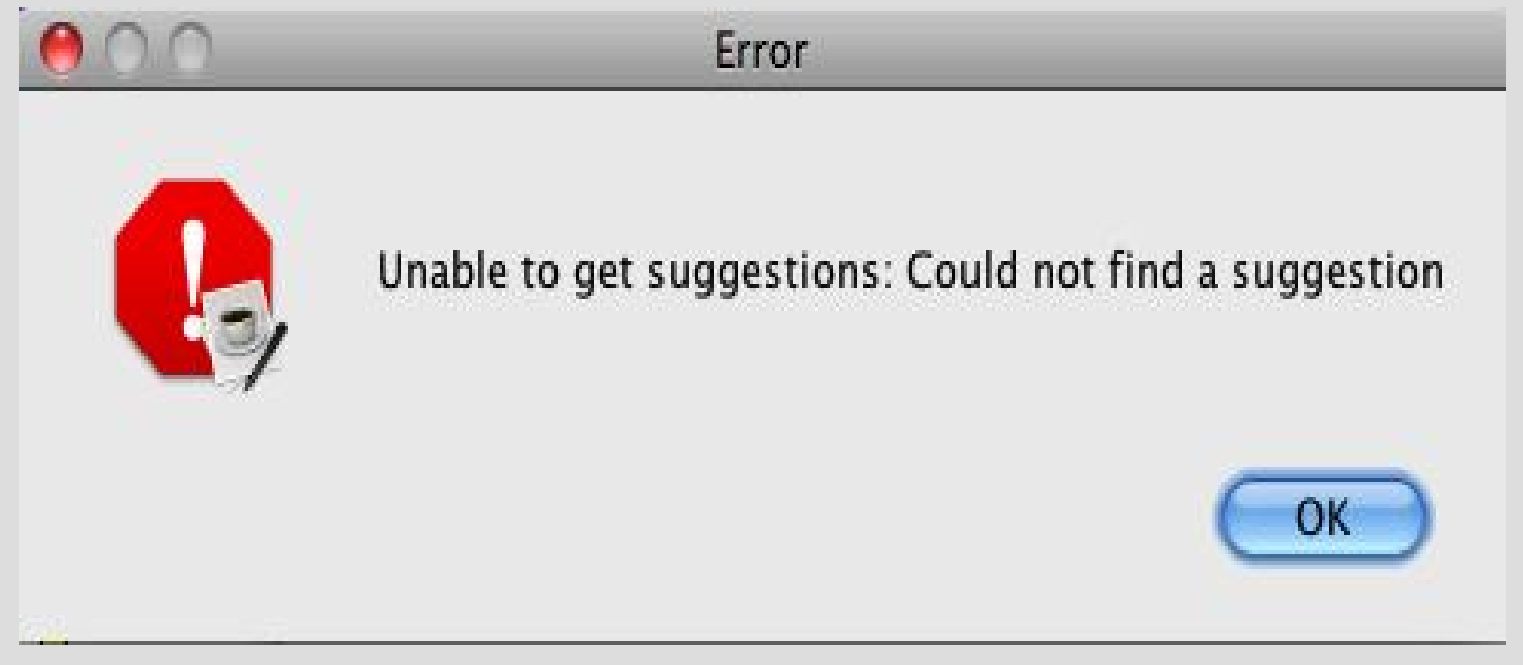




\section{Which are stable text mining solution for biologists.
Wuestions Tags Users Badges}

I am working on integrating pathway relations into a pathway diagram. I have two text mining solutions that I use to

find interactions. I am looking for new suggestions. The idea is that I provide a pathway entity (ie. gene/protein,

6 metabolite) and all relations based on the literature are returned.

text-mining interaction pathway

W edit|rollback|close | delete| flag

\section{edited Mar 1 at 7:28}

2 asked Feb 26 at 21:08

it: Andra Waagmeester

. $1,709 * 1 \bullet 13$

tag

\section{tagged \\ pathway $\times 25$ \\ interaction $\times 17$ \\ text-mining $\times 13$ \\ asked \\ 3 months ago}

viewed

284 times

latest activity

3 months ago works decently, the second has issues with performance and accessibility. - Andra Waagmeester Feb 27 at $9: 41$

add comment

\section{Answers}

oldest

newest votes

You might want to take a look at STITCH. It is a database that allows you to query with a gene/protein or a small molecule (e.g. a metabolite) and retrieve the interaction partners. A very large part of the evidence in STITCH

5 comes from text mining, and if you really want to, you can turn off all the other evidence types to exclusively get the results obtained through text mining.

Edit:

If you are using Whatizit, I would suggest that you take a look at the REST API of Reflect. It is designed for speed, so you do not need to worry about hammering the API. If you send too many requests at the same time, some of them will simply be rejected and you will get an error code back.

Could you please tell us which are the two text mining solutions that you already have? Lars Juhl Jensen Feb 27 at 7:57
Ask Question 


\section{BioStar: A question and answer site for bioinformatics}

www.biostars.org

earn reputation points and badges for participation

\section{BioStar}

Recent Questions

\begin{tabular}{l|l|l|l}
2 & 2 & 16 & Genomic coordinates from UCS
\end{tabular}

$4 \quad 6 \quad 93$ What is your favorite wiki use case in bioinformatics?

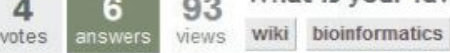

\begin{tabular}{l|l|ll}
3 & 4 & 75 & downloading fasta files
\end{tabular}

votes answers views genome fasta

1011 Velvet: retain read names in afg file

vote answers views velvet 454 afg ace reads

84206 What NGS read simulators are available for paired-end data?

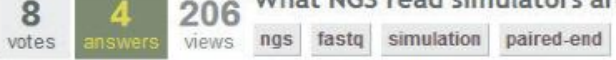

$16 \quad 12 \quad 734$ What tools/libraries do you use to visualize genomic feature data?

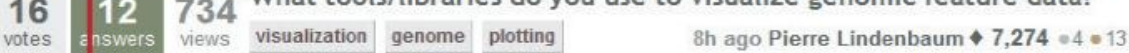

$4 \quad 1 \quad 37$ How to transform microarray data to adjust for batch effects

votes nswer views modeling batch-effect data-transformation $8 \mathrm{~h}$ ago Daniel Swan $42,669 * 1 \cdot 3$ • 14

1225 Oligo design from ESTs

vote answers views olligonucleotide design microarray est 苗

question has accepted answer

\&

OpenHelix. www.openhelix.com 1-888-861-5051

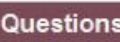

Tre

Ucsc

$29 \mathrm{~m}$ ago Pierre Lindenbaum $\$ 7,274 \bullet 4 \bullet 13$

2h ago Lee Katz $225 \cdot 6$

4h ago Lee Katz $225 \cdot 6$

$6 \mathrm{~h}$ ago jorjial 1

ask questions for the community to answer

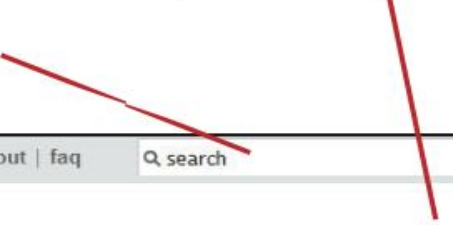

Ask Question

We're in Bootstrap Mode Senutation remuirements are relaxed while we grow the site All users can:

- Post Questions

- Post Answers

- Comment

- Create Tags

Re-Tag Questions

- Vote

Learn how you can help"

Interesting Tags

Add

Ignored Tags

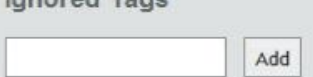

Spread the word
Tell a colleague about BioStar!
http:l//wmww.biostars.org

Subscribe: RSS feed

Admin group. Biostar Central

$9 \mathrm{~h}$ ago darked $89+1,337$ •

thenes

tags help identify topics

rate question and answers highlight/store "favorite" questions $/$

Genomic coordinates from UCSC

- have a list of gene symbols

2 APOB, TTC39B, ATF3, RGS1, LIPG,

1 am trying to get the genomic coordinates (as in bp) with $+1-5 \mathrm{~KB}$ position of these genes via UCSC TableBrowser. but I can't find the chromstart and chromEnd field in the given table. AmI missing something?

Also, please share your favorite futorial / docs that explain the schemattables in UCSC MySOL server Thanks in advance.

data-mining human-genome genome analysis ucsc

$$
\text { retag|flag }
$$

add comment

2 Answers oldest newest votes

from the table browser http://genome ucsc.edu/cal-bin/hoTables?command=start, select group= Genes 3 You'll see that knownGene is linked to $\mathrm{kg}$ Xref

hg18. kgXref. $\mathrm{kg}$ ID (via knownGene, name) Add Another Answer

Not the answer you're looking for? Browse other questions tagged data-mining human-genome genome-analysis ucsc or ask your own question.

Exponentially increasing genomes slide

I always see a slide in talks what shows an increasing number of genomes available in GenBank or other 10 database Where is this slide from? I have seen an outdated one from Genomes Online but nothing

10 How can I find this graph and cite it for my own talk?

is genome graph slides

retag| |ीag

edited Oct 19 at 10:06 (69) giovann i 3. Lee Katz $225=6$ add comment oldest newest votes has statistics at the genome, not basepair level. 


\section{BioStar \\ How do you acknowledge Biostar and its contributors in your research output?}

I am really impressed with Biostar. The quality of the answers here is often so good that it surely will have an influence. In a way Biostar extends the informal helping among peers of which gratitude is often expressed in the acknowledgments.

How does this work with Biostar. Should we acknowledge the system, all contributors, or both? Do you acknowledge Biostar in your output and if so, how?

5 biostar alt-metrics acknowledgments edit| rollback | close | delete | flag edited Apr 5 at 21:10

C.N Chris Evelo $3,794 \cdot 3 \cdot 16$

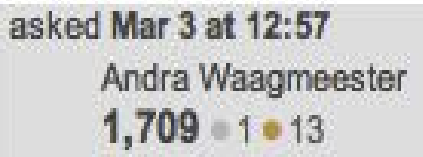

add comment

\section{Answers}

Perhaps the founders and moderators of BioStar could get together and put together a publication on BioStar and their experiences in managing it, get it published. This would provide a publication to be referenced by those who want to acknowledge the site, and would provide some direct academic benefit (a publication and citations) for at least some of those who contribute.

\section{link}

answered Mar 3 at 13:57

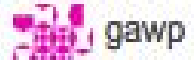

$712 \cdot 1 \cdot 8$
4 How about giving a DOI to outstanding questions? You would then have a founding paper published by the founders/moderators and a DOI to cite a specific question. This would also be an additional incentive to the biostar community. - Andra Waagmeester Mar 3 at 15:03

\section{tagged \\ biostar $\times 19$ \\ alt-metrics $\times 2$ \\ acknowledgments \\ asked \\ 3 months ago \\ viewed \\ 378 times \\ latest activity \\ 1 month ago}

Spread the wordl

Tell a colleague about BloStar!

http:lhwww.blostars.org

Subscribe: RSS feed

Admin group: Biostar Central

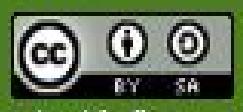

Biostar content is licensed under a 


\section{Answers}

\section{Examples}

- 2. NLM citation standard for citing blogs

- 3. A Document Object Identifier

- http://biostar.stackexchange.com/questions/6 062/ 


\section{NLM citation standard for Blogs}

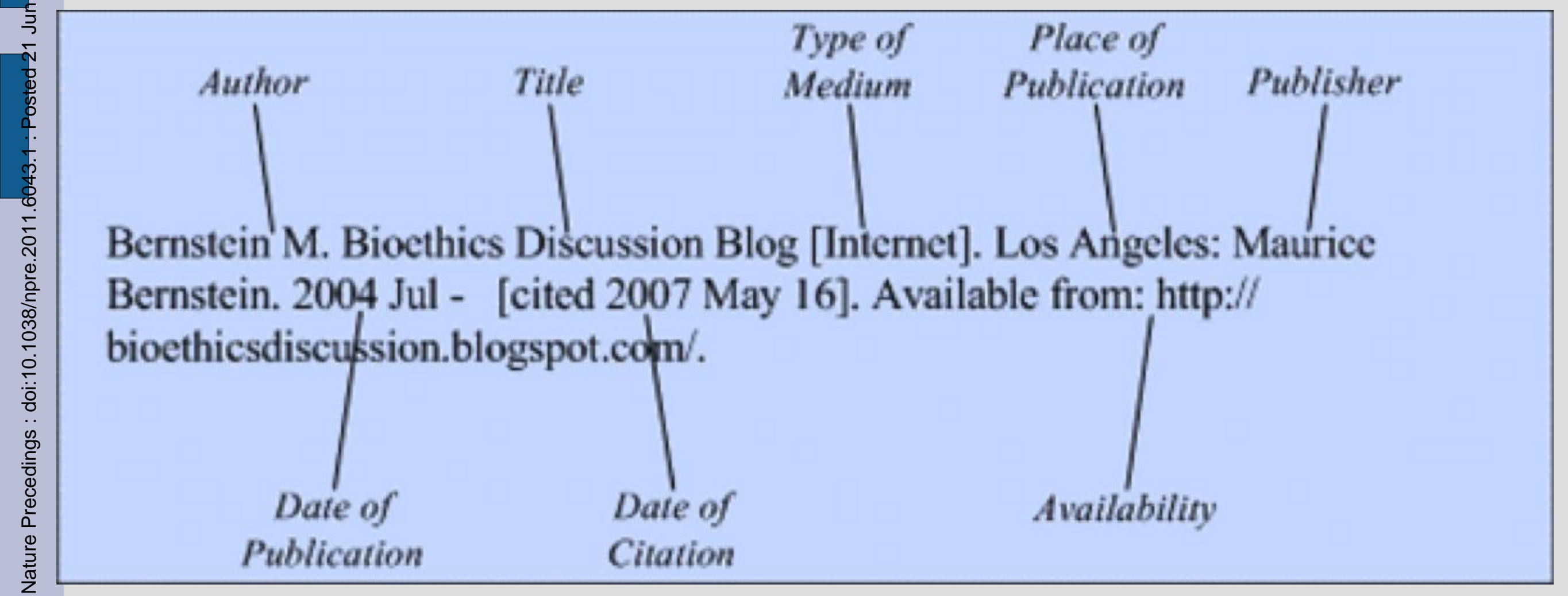




\section{Awknowledgements}

Maastricht University

- Chris Evelo

- Thomas Kelder

- Martina Kutmon

- Jahn Saito

- Martijn van lersel

USC - SF

- Alexander Pico
BIOSTAR http://biostar.

stackexchange.com

- Lars Juhl Jensen

- Casey Bergman

- Egon Willighagen

- Dominique Noel

nbIc $\equiv$

\section{Maastricht University}




\section{Acknowledgements distributed over twitter}

\section{@egonwillighagen, @larsjuhljensen You've been thanked by Andra Waagmeester at \#ib2011 for responding to BioStar query http:// bit.ly/hyWCBu \\ 3:05 AM Mar 22nd via web}

